\title{
In vitro Versuche zur antilipolytischen Wirkung von Milchsäure***
}

\author{
P. Dieterle, C. Dieterie, P. Bottermana, K. Schwarz und J. Henner****
}

II. Medizinische Klinik der Universität München (Direktor: Prof. Dr. Dr. G. BodEchtEL)

Eingegangen am 19. September 1968

The Influence of Lactic Acid on Rat Adipose Tissue Lipolysis in vitro

Summary. The influence of lactic acid on rat adipose tissuelipolysis was studied in vitro. Good correlation was found between lactic acid concentration in the medium and in. hibition of lipolysis. The correlation was equally good betwoen pyruvate production into the medium and/or lactate to pyruvate ratios and inhibition of lipolysis. So far no answer can be given whether the observed inhibition of lipolysis is a direct or indirect (pyruvate) metabolic effect of lactic acid. The possibility of an inhibition of lipolysis mediated by lactic acid during muscular exercise is discussed. vitro

Etude de l'action antilipolytique de l'acide lactique in

Résumé. L'influence de l'acide lactique sur la lipolyse a été étudiée in vitro sur le tissu adipeux du rat. L'acide lactique inhibe la lipolyse en proportion de la dose appliquée. Il y avait également une bonne corrélation entre l'inhibition de la lipolyse et la production de pyruvate dans le milieu et/ou los rapports lactate sur pyruvate. On ne peut distinguer, si l'inhibition de la lipolyse est un effet métabolique direct de l'acide lactique ou dépend de la production de pyruvate. L'inhibition de la lipolyse par l'acide lactique pendant le travail musculaire in vivo est discutée.

Zusammenfassung. Der Einfluß von Milchsäure auf die Lipolyse wird in vitro am isolierten Fettgewebe unter. sucht. Milchsäure bewirkt eine dosisabhängige Hemmung der Lipolyse. Es kann nicht entschieden werden, ob die Antilipolyse ein direkter Stoffwechseleffekt der Milchsäure ist oder nur durch diese vermittelt wird. Auffällig ist die zunehmende Hemmung der Lipolyse mit Anstieg des Redox-Potentials. Die Möglichkeit einer lactatbedingten Lipolysehemmung in vivo während muskulärer Arbeit wird diskutiert.

Key-words: Lipolysis, inhibition, lactic acid.
Das Fettgewebe als wichtiger Energiespeicher kann durch zahlreiche Substanzen und Hormone beeinflußt und gesteuert werden. Von allen Hormonen wirkt nur das Insulin zugleich antilipolytisch und lipogenetisch. Bei Stoffwechseluntersuchungen am Unterarm beobachten wir [13] nach einer kurzfristigen Arbeit einen Abfall der nicht veresterten Fettsäuren (NFS) im Blut sowie eine vermehrte Glucoseaufnahme in die Muskulatur. Sowohl die Abnahme der NFS als auch die erhöhte Glucoseaufnahme entsprechen Effekten, die man durch Insulin erzielen kann. Bei Muskelarbeit ist jedoch eher ein Abfall der Insulinkonzentration im Blut als ein Anstieg beobachtet worden [12, 32]. Berücksichtigt man diese Befunde, dann dürften die durch Muskelarbeit hervorgerufenen Stoffwechseländerungen nicht Ausdruck eines Insulineffektes sein, sondern müßten eine andere Ursache haben.

Schon lange ist ein Faktor mit insulinähnlicher Wirkung diskutiert worden, der vom arbeitenden Muskel freigesetzt wird. [20, 34]. Für seine Existenz liegen auch experimentelle Daten vor [34], wenngleich bislang eine Identifizierung nicht gelungen ist. Bei Muskelarbeit steigt als quantitativ wichtigstes Stoffwechselprodukt die Milchsäure im Blut an. IssEKuTz u. Mitarb. [24] brachten bereits 1962 den Abfall der

\footnotetext{
* Herrn Prof. Dr. Dr. Gustay Bodechtel zum 70. Geburtstag in Verehrung gewidmet.

** Mit Unterstützung der Deutschen Forschungsgemeinschaft.

*** Technische Assistenz.
}

NFS mit dem bei Arbeit vermehrt anfallenden Lactat in Zusammenhang und konnten in vivo an Hunden ihre Vermutung durch Lactatinfusionen untermauern. Die Autoren [24] konnten aber nicht entscheiden, ob es sich bei dem Abfall der NFS nur um eine kompetitive Verdrängung der NFS aus ihrer Bindung an Albumin oder um einen echten Stoffwechseleffekt handelt. Zur Klärung dieser Frage wählten wir das in vitro-System, in dem man messen kann, ob Milchsäure eine dem Insulin vergleichbare Wirkung am Fettgewebe entfaltet.

\section{Material und Methodik}

Die epididymalen Fettkörper junger $105-120 \mathrm{~g}$ schwerer Ratten wurden nach Decapitation der Tiere entnommen. Die Ratten waren vorher ad libitum mit AltrominPellets gefüttert worden. Pro Versuch mit je 12 Ansätzen wurden 12 Ratten verwandt. Die Fettkörper jeder Seite wurden in $\mathbf{6}$ etwa gleich große Stücke geteilt und derart alternierend in die Inkubationsgefäße verteilt, daß sich in jedem Ansatz von jeder Ratte Fettgewebsteilchen sowohl des rechten als auch des linken Fettanhanges befanden. Die Inkubation erfolgte nach Begasung mit Carbogen in Warburg-Gefäßen, die $5 \mathrm{ml}$ eines albuminhaltigen Krebs-Ringer-Bicarbonat-Puffers mit einem $\mathrm{pH}$ von 7.3 bis 7.4 enthielten. Nach CAMpBes et al. [6] teilweise entfettetes Rinderalbumin wurde in einer Konzentration von $5 \mathrm{~g} / 100 \mathrm{ml}$ Puffer zugegeben. Diesem Medium wurde in steigender Konzentration Milchsäure zugesetzt. Es wurde streng darauf geachtet, daß der $\mathrm{pH}$-Wert unter Zugabe von $30 \%$ iger $\mathrm{NaOH}$ exakt auf einem Wert von $\mathbf{7 . 3}$ bis 7.4 gehalten wurde. In einer weiteren Versuchsserie wurde dem Puffer-Medium zusätzlich Glucose in 
einer Konzentration von $200 \mathrm{mg} \%$ zugefügt. Alle Untersuchungen wurden als Doppelbestimmungen in jeweils 2 Ansätzen ausgeführt. Die Inkubationsdauer betrug $2 \mathrm{Std}$, die Schüttelfrequenz etwa 100 bis $120 / \mathrm{min}$. Vor und nach Inkubation wurde der Gehalt des Puffers an Glucose, Lactat, Pyruvat und Glycerin enzymatisch $[3,18,22,28]$ mit Boehringer-Test-Kombinationen, der Gehalt an NFS in einer Modifikation [14] nach DUNCOMBE [15] colorimetrisch bestimmt.

\section{Ergebnisse}

Die Befunde über den Einfluß von Milchsäure auf die Lipolyse sind in Tabelle 1 und in der Abb. 1 zusammengestellt. Die Spontanlipolyse im KRB-Puffer, gemessen an der Freisetzung von NFS und Glycerin pro $1 \mathrm{~g}$ Fettgewebe and $2 \mathrm{Std}$ wurde in Tabelle $1 \mathrm{mit}$
Medium annähernd parallel und waren nur durch die glucosebedingte Antilipolyse gegeneinander verschoben. Diese Befunde decken sich weitgehend mit Ergebnissen von BJöRNToRP [2]

Mit steigender Lactatkonzentration im Medium kam es zu einer zunehmenden Lactataufnahme durch das Fettgewebe. Gleichzeitig stiegen die Pyruvatabgabe und der Lactat-Pyruvat-Quotient bei steigendem Lactatangebot im Inkubationsmedium kontinuierlich an (Tabelle 2).

Die Glucoseaufnahme war nur bei hohen Lactatkonzentrationen über $60 \mathrm{mg} \%$ im Puffer-Medium gegenüber der Glucoseaufnahme im lactatfreien Medium signifikant erhöht.

Tabelle 1. Einfluß von Milchsäure auf die Lipolyse

Die Spontanlipolyse (Freisetzung von NFS und Glycerin) von $1 \mathrm{~g}$ Fettgewebe pro 2. Std in reinem KRB-AlbuminPuffer wurde mit 100 angegeben und die übrigen Werte dazu in Prozent \pm 1 s ausgedrückt. Die Versuche wurden sowohl im glucosefreien (Spalte a)) als auch im glucosehaltigen (Spalte b)) Medium durchgeführt

\begin{tabular}{|c|c|c|c|c|c|c|c|}
\hline & & \multicolumn{6}{|c|}{$\begin{array}{l}\text { Lactat im Medium } \\
\mathrm{mg} \%\end{array}$} \\
\hline & & 0 & 10 & 20 & 40 & 60 & 100 \\
\hline \multirow[t]{2}{*}{ NFS } & \multirow{4}{*}{$\begin{array}{l}\text { a) } \\
(n=6) \\
\text { b) } \\
(n=8) \\
\text { a) } \\
(n=6) \\
\text { b) } \\
(n=8)\end{array}$} & 100 & $74.0 \pm 18.0$ & $52.3 \pm 17.2$ & $13.2 \pm 7.6$ & $-7.2 \pm 10.7$ & $-6.8 \pm 11.6$ \\
\hline & & 100 & $69.4 \pm 1.1$ & $39.5 \pm 16.3$ & $15.2 \pm 10.0$ & $3.4 \pm 7.3$ & $-8.8 \pm 7.2$ \\
\hline \multirow[t]{2}{*}{ Glycerin } & & 100 & $83.8 \pm 9.7$ & $63.7 \pm 14.4$ & $49.7 \pm 2.8$ & $33.5 \pm 2.2$ & $26.6 \pm 8.5$ \\
\hline & & 100 & $76.1 \pm 9.7$ & $65.4 \pm 10.8$ & $44.6 \pm 4.1$ & $37.3 \pm 3.6$ & $30.0 \pm 1.6$ \\
\hline
\end{tabular}

Tabelle 2. Einfluß steigender Milchsäurekonzentrationen auf die Pyruvatbildung und den Lactat-Pyruvat-Quotienten Die Pyruvatproduktion $t 1 \mathrm{~s}$ wurde auf $1 \mathrm{~g}$ Fettgewebe pro $2 \mathrm{Std}$ sowohl im glucosefreien (Spalte a)) als auch im glucosehaltigen Medium (Spalte b)) berechnet. Mit steigenden Lactatkonzentrationen kommt es zu einer vermehrten Pyruvatbildung und zu einem Anstieg des Lactat-Pyruvat-Quotienten

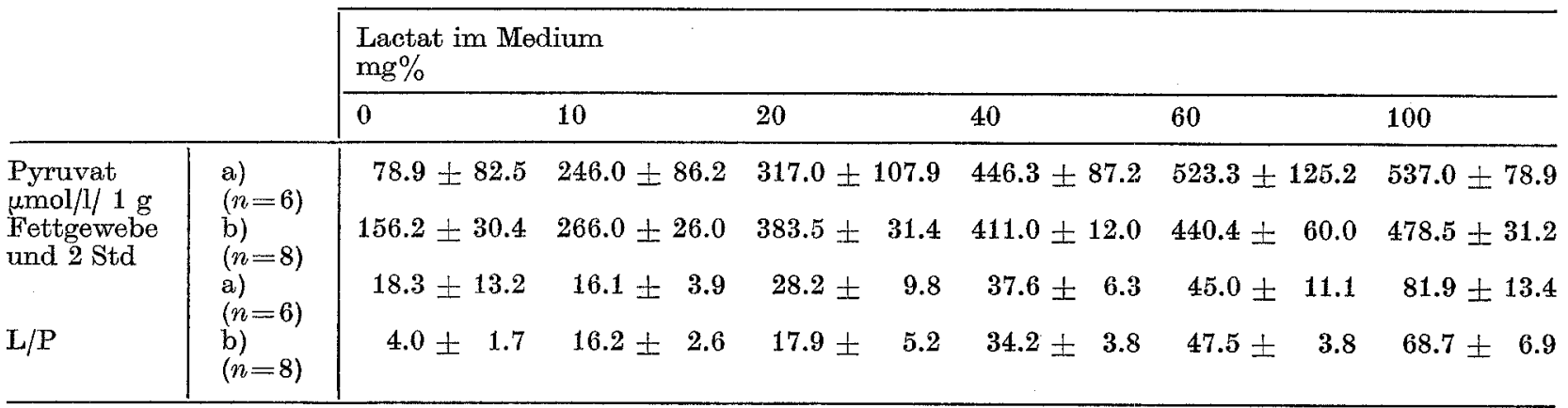

100\% eingesetzt und alle weiteren Ergebnisse prozentual dazu angegeben. Die Abgabe von NFS und Glycerin aus dem Fettgewebe verringerte sich mit steigender Konzentration von Milchsäure im Medium.

Glucosezusatz zum Puffermedium bewirkte per se eine deutliche Hemmung der Lipolyse. Diese glucosebedingte Hemmung der Lipolyse wurde durch Milchsäurezusatz wesentlich verstärkt. Die Kurven der durch Milchsäure hervorgerufenen Hemmung der Lipolyse verliefen im glucosehaltigen und glucosefreien

\section{Diskussion}

Milchsäure bewirkt in steigender Konzentration im Inkubationsmedium eine zunehmende Hemmung der Lipolyse. Dieser antilipolytische Effekt ist gleichermaBen anhand der Abnahme der NES-Freisetzung als auch der Glycerinabgabe zu beobachten (Korrelationskoeffizient $r_{\mathrm{NFS} / \mathrm{Glycerin}}: r=0.97, p<0.001$, Regressionsgerade $\left.b_{\mathrm{yx}}: 1.78\right)^{\mathbf{1}}$. Daraus ergibt sich, daß die von

\footnotetext{
1 Über ähnliche Ergebnisse berichtete BJörntoRP [2].
} 
IssekUTz et al. [24] diskutierte Möglichkeit einer kompetitiven Verdrängung der NFS aus der Albuminbindung auszuschließen ist. Würde es sich um eine einfache Verdrängung der NFS von ihrem Trägerprotein durch Milchsäure handeln, müßte die Glycerinkonzentration unbeeinflußt bleiben. Die gleichsinnige $A b$ nahme der NFS und des Glycerins läßt vielmehr den Schluß zu, daß die Triglyceridhydrolyse durch Milchsäure gehemmt wird. Es erhebt sich jedoch die Frage, ob die von uns beobachteten Stoffwechseleffekte eine direkte Folge der Milchsäure sind oder nur durch diese vermittelt werden. Während sich die NFS und der Glyceringehalt bei steigender Lactatkonzentration im Medium nach anfänglich annähernd linearem Abfall asymptomatisch einem Endwert nähern (Abb. 1), er-

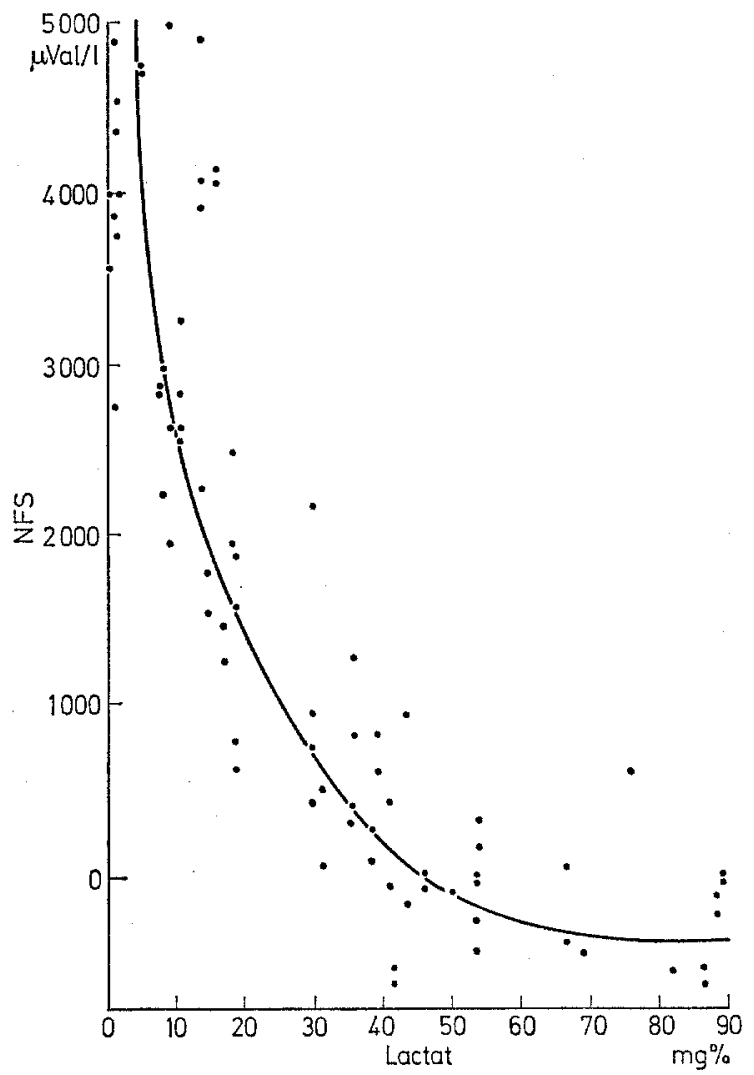

Abb. 1. Der Einfluß steigender Milchsäurekonzentration im Puffermedium auf die Abgabe der nicht veresterten Fettsäuren (NFS) des Fettgewebes: Die NFS-Abgabe wurde in $\mu$ val/L pro $1 \mathrm{~g}$ Fettgewebe und 2 Std berechnet. Mit steigender Milchsäurekonzentration kommt es zu einer zunehmenden Hemmung der Lipolyse, die sich bei hohen Lactatkonzentrationen asymptotisch einem Endwert nähert

gibt der Vergleich der NFS- und Glycerinkonzentration gegen das bei steigender Lactatzugabe vermehrt gebildete Pyruvat eine ausgezeichnete Korrelation ( $r_{\text {Glycerin }}:-0.75 ; r_{\text {NFS }}: 0.97$; siehe auch Abb. 2). Eine gleich gute Korrelation erhält man beim Vergleich der Spaltprodukte der Triglyceride und des Lactat-Pyruvat-Quotienten. Es muß deshalb die Möglichkeit disku- tiert werden, daß die Hemmung der Lipolyse von der Konzentration der Brenztraubensäure oder vom Lactat-Pyruvat-Quotienten, also vom Redox-Potential, abhängig ist. Aus Untersuchungen von BuckLE et al. [4] ist bekannt, daß steigende Pyruvatzugabe zum Inkubationsmedium eine zunehmende Hemmung der Lipolyse bewirkt. Allerdings lagen die Brenztraubensäurekonzentrationen bei BUCKLE [4] um eine Zehnerpotenz höher als die in unseren Versuchen beobachteten Pyruvatspiegel.

Während Lactat am isolierten Fettgewebe eine dem Insulin vergleichbare Hemmung der Lipolyse hervorrief, wurde die Glucoseaufnahme nur bei hohen Lactatkonzentrationen über $60 \mathrm{mg} \%$ im Medium stimuliert.

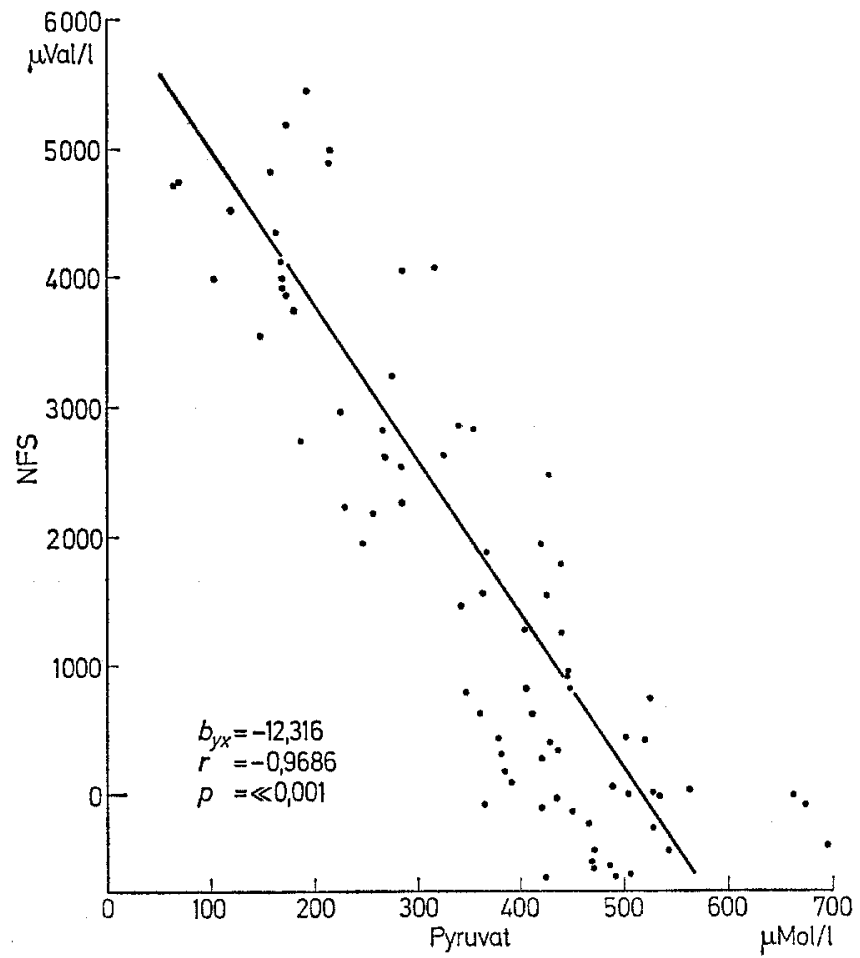

Abb. 2. Abhängigkeit der NFS-Abgabe aus dem Fettgewebe von der Pyruvatfreisetzung: Die Werte wurden auf $1 \mathrm{~g}$ Fettgewebe pro $2 \mathrm{Std}$ berechnet. Mit zunehmender Pyruvatfreisetzung kommt es zu einer zunehmenden Hemmung der Lipolyse. Es ergibt sich eine ausgezeichnete Korrelation

Schließlich erhebt sich die Frage, ob unsere am isolierten Rattenfettgewebe gewonnenen in vitro Ergebnisse auf in vivo-Verhältnisse beim Menschen übertragbar sind. Bei Muskelarbeit kommt es einerseits zu einem beträchtlichen Lactatanstieg im Blut. Andererseits ist aus vielen Untersuchungen bekannt, daß die NFS anfänglich im Blut deutlich abfallen, um erst bei länger dauernder Arbeit über den Ausgangsspiegel anzusteigen [7, 10, 23-25, 27, 29]. Ein Abfall des NFSBlutspiegels kann entweder die Folge einer verminderten Lipolyse oder einer erhöhten Aufnahme ins Ge- 
webe oder aber auch Folge beider Prozesse zugleich sein. Untersuchungen mit markierten Fettsäuren zeigten eindeutig, daß die Fettsäureoxydation während. einer Arbeit um ein Vielfaches gegenüber Ruhebedingungen ansteigt $[5,16,17,21]$. Der Abfall der NFS im Blut wäre durch vermehrte Ausschöpfung durch die Muskulatur infolge des erhöhten Energiebedarfse während der Arbeit zu erklären. Dem steht jedoch entgegen, daß der NFS-Abstrom ins Gewebe eine Funktion des NFS-Spiegels im Blut ist [17, 33] und bei niedrigem Fettsäurespiegel im Blut nur wenig Fettsäuren abgegeben werden. Außerdem liegen Ergebnisse vor, nach denen die erhöhte Fettverbrennung während der Arbeit zu einem Teil aus muskeleigenen Fettreservoirs gedeckt werden kann $[26,31]$.

In diesem Zusammenhang sind in vivo-Versuche am. ruhenden Hund und am Menschen von Bedeutung [13, $19,24,30]$, bei denen ein ähnlicher NFS-Abfall durch Lactat-Infusionen hervorgerufen werden konnte, wie er initial bei Muskelarbeit gesehen wird

CoBB und JoHrson [11] führten bei physisch trainierten und untrainierten Personen Belastungen durch. Die trainierten reagierten auf die Belastung mit einem geringeren Anstieg des Lactats als die untrainierten. Andererseits war bei den untrainierten Personen mit höheren Blut-Lactatspiegeln der initiale Fettsäureabfalle ausgeprägter als bei den Personen mit geringerem Lactatanstieg.

Diese in vivo gewonnenen Ergebnisse sprechen in Zusammenhang mit unseren Befunden dafür, daß neben einer möglichen vermehrten NFS-Ausschöpfung aus dem Blut während der Arbeit auch das dabei anfallende Lactat einen Einfluß auf die Lipolyse ausübt.

Dabei bleibt selbstverständlich die Frage noch offen, warum bei länger andauernder Arbeit trotz der anhaltenden Lactatproduktion die NFS-Spiegel im Blut über die Ausgangswerte ansteigen. Dieses Pro. blem sollte durch weitere Untersuchungen bearbeitet werden.

Fräulein R. ERMLER und Fräulein U. HranderKotr sind wir für ihre wertvolle Mitarbeit zu großem Dank verpflichtet.

\section{Literatur}

1. Basu, A., R. Passmore, and J.A. Strong: The effect of exercise on the level of non-esterified fatty acids in the blood. Quart. J. exp. Physiol. 45, 312-317 (1960).

2. Buörntorp, P.: The effect of lactic acid on adipose tissue metabolism in vitro. Acta med. scand. 178, $253-$ 255 (1965).

3. BÜCHer, Th., R. Czok, W. Lampraecht u. E. Latzko: Pyruvat. In: Bergmeyer, U., edit. Methoden der enzymatischen Analyse, S. 253-259. Weinheim/Bergstraße: Verlag Chemie 1962.

4. Buckie, R.M., D. RubinsteIn, E.E. MoGarky, and J.C. BECK: Factors influencing the relase of free fatty acids from rat adipose tissue. Endocrinology 69, 1009-1015 (1961).

5. BÜHRING, H., u. F. HoCkeMTEYer: Modelluntersuchungen zum Energiestoffwechsel des Muskels. TI. Die Oxydation von Fettsäure und Glucose sowie die
Milchsäurebildung im ruhenden und arbeitenden Skelettmuskel in vitro. Biochem. Z. 346, 50-59 (1966).

6. Campbeli, J., A.D. Martucci, and G.R. GReen: Plasma albumin as an acceptor of free fatty acids. Biochem. J. 93, 183-189 (1964).

7. CARlson, L.A., L.-G. EkgluUnd, and L. Orö: Studies on blood lipids during exercise. TV. Arterial concentration of plasma free fatty acids and glycerol during and after prolonged exercise in normal men. J. Lab. clin. Med. 61, 724-729 (1963).

8. --, and B. Peknow: Studies on blood lipids during exercise. I. Arterial and venous plasma concentration of unesterified fatty acids. J. Lab. clin. Med. 53, 833841 (1959).

9. - - Studies on blood lipids during exercise. II. The arterial plasma free fatty acids concentration during and after exercise and its regulation. J. Lab. clin. Med. $58,673-681$ (1961).

10. Cartström, S.: Studies on fatty acid metabolism in diabetics during exercise. I. Plasma free fatty acid concentration in juvenile newly diagnosed diabetics during exercise. Acta med. scand. 181, 609-621 (1967).

11. CoBs, L.A., and W.P. JoHnson: Hemodynamic relationships of anaerobic metabolism and plasma free fatty acids during prolonged, strenuous exercise in trained and untrained subjects. J. clin. Invest. 42, $800-810$ (1963).

12. Cochran, B., E.P. Marbadr, R. Poucher, T. SteinBERG, and G. GWTruP: Effect of acute muscular exercise on serum immunoreactive insulin concentration. Diabetes 15, 838-841 (1966).

13. DTETERLe, $P$. et al.: unveröffentlicht.

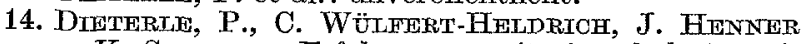
u. K. SoHwarz: Erfahrungen mit einer kolorimetrischen Methode zur Bestimmung der nicht veresterten Fettsäuren im Serum. Z. klin. Chem. Biochem. 5, $153-155$ (1968).

15. Duncomes, W.G.: The colorimetric micro-determination of non-esterified fatty acids in plasma. Clin. chim. Acta 9, 122-125 (1964).

16. Friedberg, S.J., W.R. Harlai, D.L. Trout, and E.H. EsTes: The effect of exercise on the concentration and turnover of plasma nonesterified fatty acids. J. clin. Invest. 39, 215-220 (1960).

17. Fritz, I.B., D.G. Davis, R.H. Holtroop, and H. DUNDEE: Fatty acid oxidation by skeletal muscle during rest and activity. Amer. J. Physiol. 194, 379386 (1958).

18. Fromsch, E.R., A.E. REnold, and N.B. MoWILLIAMS: Specific enzymatic determination of glucose in blood and urine using glucose oxidase. Diabetes $\mathbf{5}$, $1-6(1956)$.

19. Gotd, M., H.I. Mirlet, B. Isseku'tz, and J.J. SPITzER: Effect of exercise and lactic infusion on individual free fatty acids of plasma. Amer. J. Physiol. 205, $902-904$ (1963).

20. Gotdstern, M.S.: Muscular exercise and subsequent glucose utilization. In B.S. LAtiBEL and G.A. WRENSHALL: "On the nature and treatment of Diabetes". Amsterdam: Excerpta med. Foundation 1965.

21. Haver, R.J., A. NAmark, and Ch. F. BorchgreVINK: Turnover rate and oxidation of free fatty acids of blood plasma in man during exercise, Studies during continuous infusion of palmitate-1-C14. J. clin. Invest. 42, 1054-1063 (1963).

22. HoHoRst, H. : L- $(+)$-Lactat. Bestimmung mit Lactat. Dehydrogenase, in Bergmeyer, U., edit. Methoden der enzymatischen Analyse, S. 266. Weinheim/Berg. straße: Verlag Chemie 1962.

23. Issekutz, B., W.M. Bortz, H.I. Mrluer, and A. Wroldsen: Plasma free fatty acid response to exercise in obese humans. Metabolism 16, 492-502 (1967). 
24. - , and H. ML工工ER: Plasma free fatty acids during exercise and the effect of lactic acid. Proc. Soc. exp. Biol. (N.Y.) 110, 237-239 (1962).

25. - H.T. MrLLer, and K. Rodaht: Effect of exercise on FFA metabolism of pancreatectomized dogs. Amer. J. Physiol. 205, 645-650 (1963).

26. - - P. PAUL, and K. RODAHL: Source of fat oxidation in exercising dogs. Amer. J. Physiol. 207, 583589 (1964).

27. Kumin, R.F., W.G. Troyer, K.W. Back, Th. C. Hood, and M.D. Bogdonofr : Lipid mobilization in lean and obese subjects. Ann. N.Y. Acad. Sci. 131, $662-672(1965)$.

28. Krevtz, F.H.: Fnzymatische Glycerinbestimmung. Klin. Wschr. 40, 362-363 (1962).

29. Milder, H., B. Issekutz, and K. RodahL: Effect of exercise on the metabolism of fatty acids in the dogs. Amer. J. Physiol. 205, 167-172 (1963).

30. Mililer, H.I., B. Issekutz, P. PAUL, and K. Rodahl: Effect of lactic acid on plasma free fatty acids in pancreatectomized dogs. Amer. J. Physiol. 207, 12261230 (1964).
31. Neptune, E.M., H.C. Sudduth, and D.R. Foreman: Labile fatty acids of rat diaphragm muscle and their possible role as the major substrate for maintenance of respiration. J. biol. Chem. 234, 1659-1660 (1959).

32. NIKkILÄ, E.A., M.R. TASkINEN, T. MtentineiN, R. Pelikonen, and $H$. Poppius: Effect of muscular exercise on plasma insulin fluxes. Stockholm: 6. Congr. Internat. Diabetes Fed. 1967.

33. SPITzer, J.J., and M. GoLD : Free fatty acid metabolism by skeletal muscle. Amer. J. Physiol. 206, 159162 (1964).

34. WerThermiger, H.E., and E. Havivi: The in vitro release of a product of muscular activity which increases the transport of sugars into diaphragm. Harefuah 62, 251-254 (1962), zit. nach GoudSIEIN [20].

Dr. P. Dieterte cand. med. C. Dietererte Dr. P. BoTTERMANN Prof. Dr. K. Schwarz II. Medizinische Klinik der Universität 8 München 15 Ziernssenstraße 1 